\title{
FluxnetLSM R package (v1.0): A community tool for processing FLUXNET data for use in land surface modelling
}

Ukkola A.M. ${ }^{1}$, Haughton N. ${ }^{1}$, De Kauwe M.G. ${ }^{2}$, Abramowitz G. ${ }^{1,3}$, Pitman A.J. ${ }^{1,3}$

${ }^{1}$ ARC Centre of Excellence for Climate System Science, University of New South Wales, Sydney, NSW 2052, Australia

${ }^{2}$ Macquarie University, Sydney, NSW 2109, Australia

${ }^{3}$ Climate Change Research Centre, University of New South Wales, Sydney, NSW 2052, Australia

\section{Supplementary Material}

This supplementary material includes Supplementary Tables 1 and 2. An example script for processing a single site is provided in section S.1. 


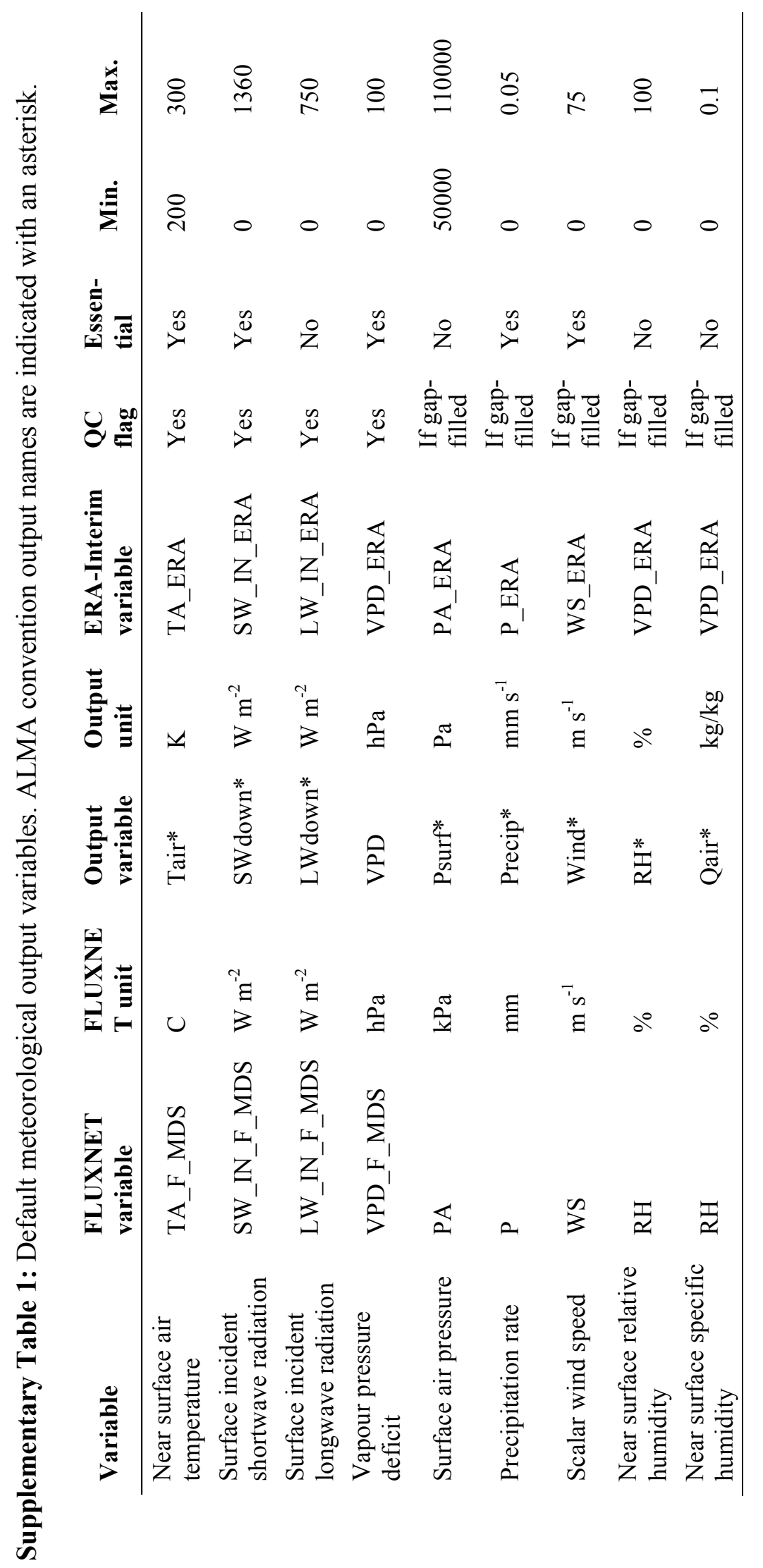




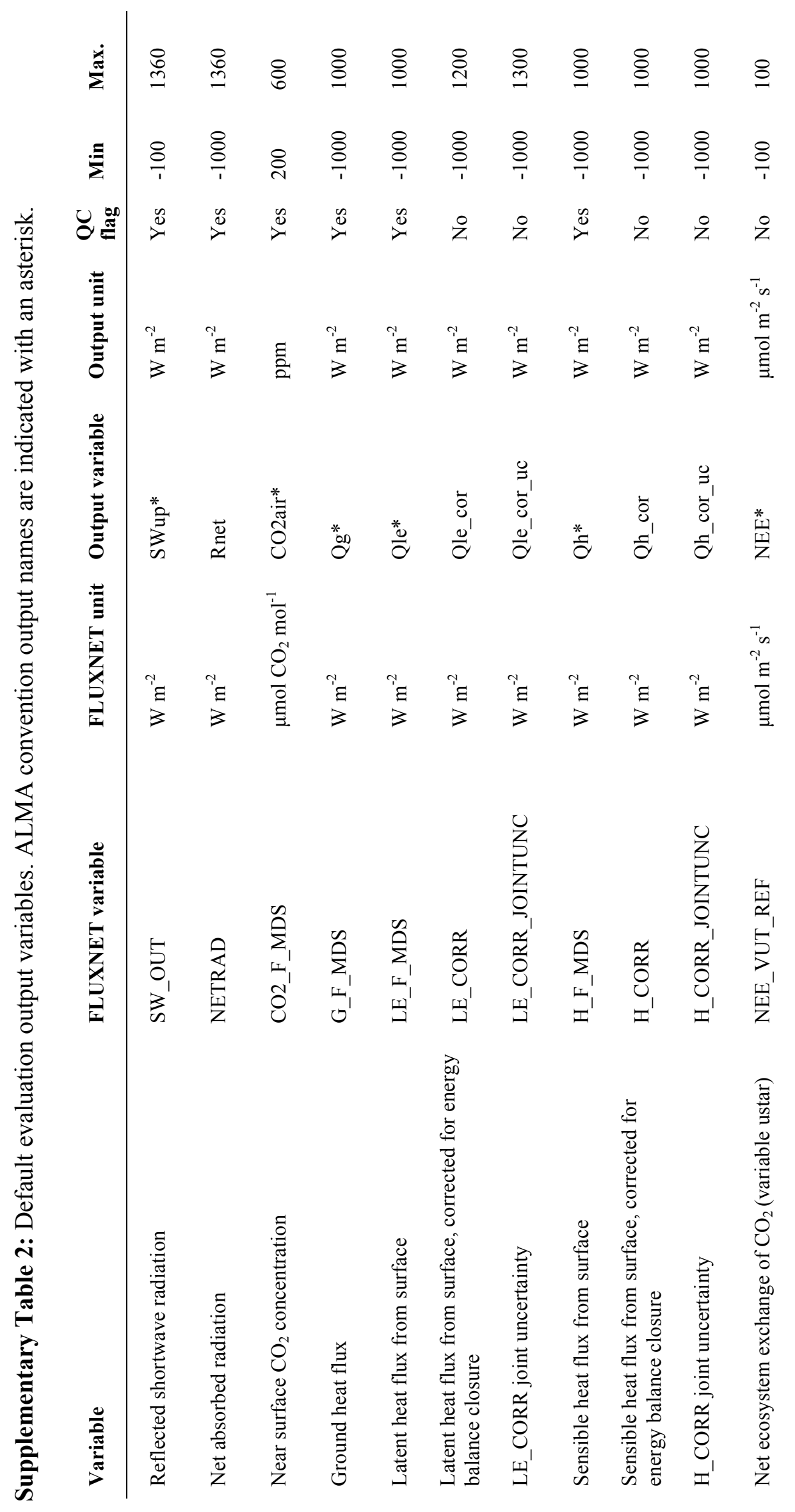




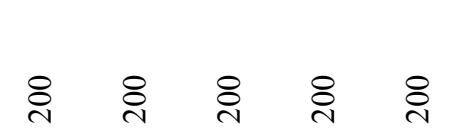

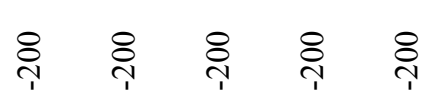

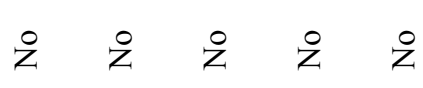

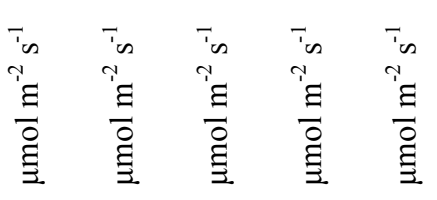

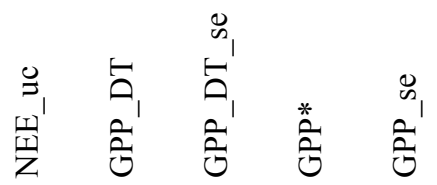

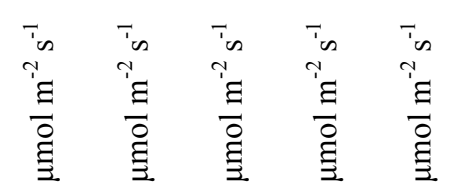

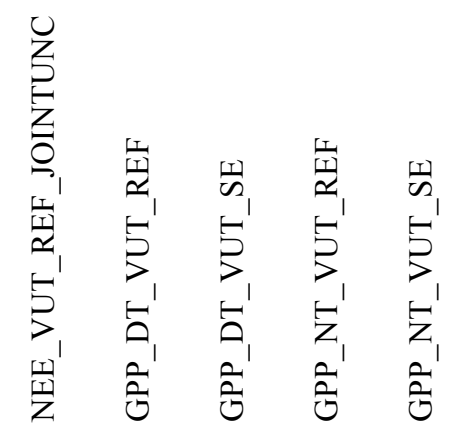

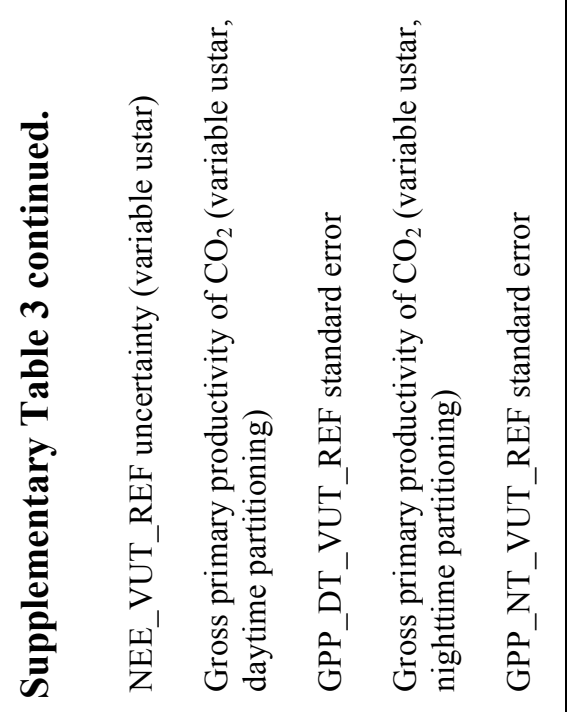




\section{S.1 Example usage of FluxnetLSM}

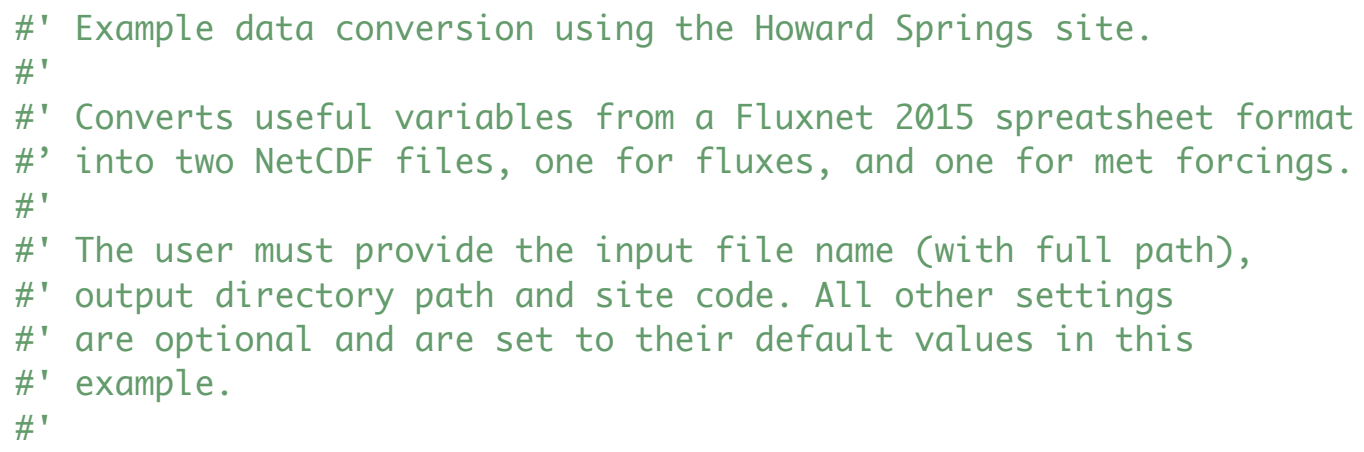


\#What percentage of time steps allowed to be missing \#or gap-filled in any given year? And minimum number of consecutive \#years to process. Note: Always checks for missing values. If no \#gapfilling thresholds are set, will not check for gap-filling.

missing <- 15 \#max. percent missing (must be set)

gapfill_all <- 20 \#max. percent gapfilled (optional)

gapfill_good <- NA \#max. percent good-quality gapfilled (optional, \#ignored if gapfill_all set)

gapfill_med <- NA \#max. percent medium-quality gapfilled (optional, \#ignored if gapfill_all set)

gapfill_poor <- NA \#max. percent poor-quality gapfilled (optional, \#ignored if gapfill_all set)

min_yrs <- 2 \#min. number of consecutive years

\#Should code produce plots to visualise outputs? (set to NA if not \#desired)(annual: average monthly cycle; diurnal: average diurnal \#cycle by season; timeseries: 14-day running mean time series) plot <- c("annual", "diurnal","timeseries")

\#\#\#\#\#\#\#\#\#\#\#\#\#\#\#\#\#\#\#\#\#\#

\#\#\#--- Run analysis ---\#\#\#

\#\#\#\#\#\#\#\#\#\#\#\#\#\#\#\#\#\#\#\#\#\#

convert_fluxnet_to_netcdf(infile=infile, site_code=site_code, out_path=out_path, ERA_file=ERA_file, ERA_gapfill=ERA_gapfill, datasetname=datasetname, datasetversion=datasetversion, missing=missing, gapfill_all=gapfill_all, gapfill_good=gapfill_good, gapfill_med=gapfill_med, gapfill_poor=gapfill_poor, include_all_eval=TRUE, min_yrs=min_yrs, plot=plot) 